Bull. Austral. Math. Soc.

$60 \mathrm{G} 42,60 \mathrm{G} 46$

VOL. 45 (1992) [43-52]

\title{
ON THE DUALITY OF SOME MARTINGALE SPACES
}

\author{
N.L. Bassily and A.M. Abdel-Fattah
}

Fefferman has proved that the dual space of the martingale Hardy space $H_{1}$ is the $B M O_{1}$-space. Garsia went further and proved that the dual of $H_{p}$ is the so-called martingale $K_{p}$-space, where $p$ and $q$ are two conjugate numbers and $1 \leqslant p<2$.

The martingale Hardy spaces $H_{\$}$ with general Young function $\Phi$, were investigated by Bassily and Mogyoródi. In this paper we show that the dual of the martingale Hardy space $H_{\ddagger}$ is the martingale Hardy space $H_{\Phi}$ where $(\Phi, \Psi)$ is a pair of conjugate Young functions such that both $\Phi$ and $\Psi$ have finite power. Moreover, two other remarkable dualities are presented.

\section{BASIC NOTATIONS AND DEFINITIONS}

Let $X \in L^{1}(\Omega, A, P)$ be a random variable defined on the probability space $(\Omega, A, P)$ and consider the regular martingale

$$
X_{n}=E\left(X \mid F_{n}\right), \quad n \geqslant 0,
$$

where $\left\{F_{n}\right\}, n \geqslant 0$, is an increasing sequence of $\sigma$-fields of events such that

$$
F_{\infty}=\sigma\left(\bigcup_{n=0}^{\infty} F_{n}\right)=A .
$$

We suppose that $X_{0}=0$ almost surely. We denote by $d_{0}=0, d_{1}, d_{2}, \ldots$ the difference sequence corresponding to the martingale $\left(X_{n}, F_{n}\right)$.

The $K_{p}$-spaces were investigated by Garsia (see [2]).

In [3] we generalised this notion. Consider a pair $(\Phi, \Psi)$ of conjugate Young functions and let

$$
\mu_{X}^{(\Phi)}=\left\{\gamma: \gamma \in L^{\Phi}, E\left(\left|X-X_{n-1}\right| \mid F_{n}\right) \leqslant E\left(\gamma \mid F_{n}\right) \text { almost surely } \forall n \geqslant 1\right\}
$$

We say that $X \in K_{\Phi}$ if the set $\mu_{X}^{(\Phi)}$ is not empty. In this case we define

$$
\|X\|_{K_{\Psi}}=\inf _{\gamma \in \mu_{X}^{(\Phi)}}\|\gamma\|_{\Phi}
$$

Received 14 January 1991

Copyright Clearance Centre, Inc. Serial-fee code: 0004-9729/92 \$A2.00+0.00. 
where $\|\cdot\|_{\Phi}$ denotes the Luxemburg norm in the Orlicz-space $L^{\Phi}$. For the definition of the Young functions, Orlicz-spaces and Luxemburg norms we refer to [4] and [5]. It is easily proved that $\left(K_{\Phi},\|\cdot\|_{K_{\Phi}}\right)$ is a Banach space (see [3]). The space $K_{\infty}$ is the well-known $B M O_{1}$-space.

We say that the random variable $X$ belongs to the Hardy space $H_{\Phi}$ if the quadratic variation

$$
S=S(X)=\left(\sum_{i=1}^{\infty} d_{i}^{2}\right)^{1 / 2} \in L^{\Phi}
$$

It is easy to show that $H_{\Phi}$ with the norm $\|X\|_{H_{\Psi}}=\|S\|_{\Phi}$ is a Banach space (see [3]).

A Young function $\Phi$ is said to be of moderated growth if its power

$$
p=\sup _{x>0}(x \phi(x)) /(\Phi(x))
$$

is finite. Here $\phi(x)$ stands for the right-hand side derivative of $\Phi$.

\section{AUXILIARY RESULTS}

LEMMA 1. If the Young function $\Phi$ has a finite power, then $H_{\Phi} \subset K_{\Phi}$.

Proof: In fact, the Burkholder-Davis-Gundy inequality (see [6, Theorem 15.1]) guarantees that $X \in H_{\Psi}$ implies that

$$
X^{*}=\sup _{n \geqslant 1}\left|X_{n}\right| \in L^{\Phi}
$$

where $X_{n}=E\left(X \mid F_{n}\right), n \geqslant 1$.

From this for all $n \geqslant 1$ we have almost surely.

$$
E\left(\left|X-X_{n-1}\right| \mid F_{n}\right) \leqslant E\left(2 X^{*} \mid F_{n}\right)
$$

Consequently, $X \in K_{\Phi}$ with $\|X\|_{K_{\Phi}} \leqslant 2\left\|X^{*}\right\|_{\Phi}$.

The following assertion gives a sufficient condition which ensures that the martingale Hardy space $H_{\Phi}$ and the martingale space $K_{\Phi}$ coincide and the corresponding norms are equivalent.

TheOREM 1. Suppose that $\Phi$ and its conjugate $\Psi$ have finite powers $p$ and $q$ respectively. Then, the spaces $H_{\Phi}$ and $K_{\Phi}$ coincide. More precisely, there exist positive constants $c_{\Phi}^{(1)}$ and $C_{\Phi}^{(1)}$ depending only on $\Phi$ such that

$$
c_{\Phi}^{(1)}\|X\|_{K_{\Phi}} \leqslant\|X\|_{H_{\Phi}} \leqslant C_{\Phi}^{(1)}\|X\|_{K_{\Phi}}
$$


Proof: Suppose that $X \in K_{\Phi}$. Let $X_{n}=E\left(X \mid F_{n}\right), n \geqslant 1$ be the corresponding regular martingale and let us define

$$
X_{n}^{*}=\max _{1 \leqslant \ell \leqslant n}\left|X_{\ell}\right|, \quad n \geqslant 1
$$

This random variable with arbitrary constants $\beta>\alpha>0$ satisfies the inequality

$$
(\beta-\alpha) P\left(X_{n}^{*} \geqslant \beta\right) \leqslant E\left(\gamma \chi\left(X_{n}^{*} \geqslant \alpha\right)\right)
$$

where $\gamma \in \mu_{X}^{(\Phi)}$ is arbitrary and $\chi_{(B)}$ stands for the indicator of $B$.

For arbitrary $A>0$ define

$$
X_{n}^{* *}=\min \left(X_{n}^{*}, a\right) .
$$

Then $X_{n}^{* *} \in L_{\infty}$ and for arbitrary $\lambda>0$ we have

$$
\chi\left(X_{n}^{* *} \geqslant \lambda\right)= \begin{cases}0 & \text { if } \lambda>a \\ \chi\left(X_{n}^{*} \geqslant \lambda\right), & \text { if } \lambda \leqslant a .\end{cases}
$$

Consequently, since $\beta>\alpha>0$, it follows that

$$
(\beta-\alpha) P\left(X_{n}^{* *} \geqslant \beta\right) \leqslant E\left(\gamma \chi\left(X_{n}^{* *} \geqslant \alpha\right)\right)
$$

Choose $\beta=c \alpha$ where $c>1$ is a constant, and integrate the above inequality with respect to the measure $d \phi(\alpha)$ and using Fubini's theorem we get

$$
(c-1) E\left(\Psi\left(\phi\left(\frac{X_{n}^{* *}}{c}\right)\right)\right) \leqslant E\left(\gamma \phi\left(X_{n}^{* *}\right)\right) .
$$

Since $\Phi$ has finite power, then for any $c>1$ there exists a constant $A=A(c)>0$ such that

$$
\phi(c x) \leqslant A \phi(x), \quad x \geqslant 0 .
$$

From the preceding inequality we get

$$
(c-1) E\left(\Psi\left(\phi\left(\frac{X_{n}^{* *}}{c}\right)\right)\right) \leqslant A E\left(\gamma \phi\left(\frac{X_{n}^{* *}}{c}\right)\right) .
$$

Applying Young's inequality and rearranging, we have

$$
(\rho-1) E\left(\Psi\left(\phi\left(\frac{X_{n}^{* *}}{c}\right)\right)\right) \leqslant E(\Phi(\gamma / b)),
$$


where $b=(c-1) /(A \rho)$ and $\rho>1$ is arbitrary.

Let $A \uparrow+\infty, X_{n}^{* *} \uparrow X_{n}^{*}$ and by the monotone convergence theroem we have

$$
(\rho-1) E\left(\Psi\left(\phi\left(\frac{X_{n}^{*}}{c}\right)\right)\right) \leqslant E(\Phi(\gamma / b)) .
$$

Applying the so obtained inequality to the new martingale

we get

$$
\left(\frac{X_{k}}{\|\gamma\|_{\Phi}} b, F_{k}\right), \quad k=1,2, \ldots
$$

$$
(\rho-1) E\left(\Psi\left(\phi\left(\frac{X_{n}^{*}}{\rho \frac{c}{c-1} A\|\gamma\|_{\Phi}}\right)\right)\right) \leqslant 1 \text {. }
$$

Since $q$, the power of $\Psi$ is finite it follows that with $\rho=q$

$$
\left\|X_{n}^{*}\right\|_{\Phi} \leqslant q \frac{c}{c-1} A\|X\|_{K_{\Phi}}
$$

REMARK. Especially, with $\Phi(x)=x^{p} / p, p>1$, we have $\phi(x)=x^{p-1}$ and $\Psi(x)=$ $x^{q} / q, q>1$ where $1 / p+1 / q=1$. Thus, if $K \in K_{\Phi}=K_{p}$ we have

$$
\left\|X_{n}^{*}\right\|_{p} \leqslant q \frac{c^{p}}{c-1}\|X\|_{K_{p}}
$$

This is the inequality obtained by Garsia ([2, Theorem III.5.2]). The constant $c>1$ is used to optimise the coefficient on the right hand side in the preceding inequaity. The minimal value of $\left(c^{p}\right) /(c-1)$ is obtained when $c=p /(p-1)$. Thus we get

$$
\left\|X_{n}^{*}\right\|_{p} \leqslant p q^{p}\|X\|_{K_{p}} \leqslant p q e\|X\|_{K_{p}}
$$

Now, let us denote $X^{*}=\sup _{n \geqslant 1}\left|X_{n}\right|$, then by the monotone convergence theorem we have

$$
\left\|X^{*}\right\|_{\Phi} \leqslant q \frac{c}{c-1} A\|X\|_{K_{\Phi}} .
$$

We deduce that $X^{*} \in L^{\Phi}$. By the above mentioned Burkholder-Davis-Gundy inequality it follows that $X \in H_{\Phi}$ and with some $C_{\Phi}^{\prime}>0$ we have

$$
c_{\Phi}^{\prime}\|X\|_{H_{\Phi}} \leqslant\left\|X^{*}\right\|_{\Phi} \leqslant q \frac{c}{c-1} A\|X\|_{K_{\Phi}} .
$$

This proves the right hand side of our inequality.

Conversely, suppose that $X \in H_{\Phi}$, then using Lemma 1, with some constant $c_{\Phi}^{\prime \prime}>0$, we have

$$
\|X\|_{K_{\Phi}} \leqslant 2\left\|X^{*}\right\|_{\Phi} \leqslant 2 c_{\Phi}^{\prime \prime}\|X\|_{H_{\Phi}}
$$


This proves the left hand side of our inequality.

LEMma 2. Let $(\Phi, \Psi)$ be a pair of conjugate Young functions and suppose that both $\Phi$ and $\Psi$ have finite power $p$ and $q$ respectively. Then for every $X \in H_{\Phi}$ there exist positive constants $c_{\Phi}^{(2)}$ and $C_{\Phi}^{(2)}$ depending only on $\Phi$ such that the following two sided inequality holds:

$$
c_{\Phi}^{(2)} \sup _{n \geqslant 0}\left\|X-X_{n}\right\|_{\Phi} \leqslant\|X\|_{H_{\Phi}} \leqslant C_{\Phi}^{(2)} \sup _{n \geqslant 0}\|X-X\|_{\Phi}
$$

Here $X_{n}=E\left(X \mid F_{n}\right), n \geqslant 0$.

Proof: Denote $X^{*}=\sup _{n \geqslant 0}\left|X_{n}\right|$. Since $\Phi$ has finite power, then by the BurkholderDavis-Gundy inequality we have

$$
c_{\Phi}^{\prime}\|X\|_{H_{\Phi}} \leqslant\left\|X^{*}\right\|_{\Phi} \leqslant C_{\Phi}^{\prime \prime}\|X\|_{H_{\Phi}}
$$

where $c_{\Phi}^{\prime}$ and $c_{\Phi}^{\prime \prime}$ are positive constants depending only on $\Phi$. Since $\Psi$ has a finite power $q$, then using Doob's maximal inequality (see [7]) we have

$$
\sup _{n \geqslant 0}\left\|X_{n}\right\|_{\Phi} \leqslant\left\|X^{*}\right\|_{\Phi} \leqslant q \sup _{n \geqslant 0}\left\|X_{n}\right\| \text {. }
$$

Remarking that $X_{0}=0$ almost surely and that $\left\|X_{n}\right\|_{\Phi} \uparrow\|X\|_{\Phi}$ by using Jensen's inequality and by [4, Appendix (Proposition A-3-4)], (2) implies that

$$
\frac{1}{2} \sup _{n \geqslant 0}\left\|X-X_{n}\right\|_{\Phi} \leqslant \sup _{n \geqslant 0}\left\|X_{n}\right\|_{\Phi} \leqslant\left\|X^{*}\right\|_{\Phi} \leqslant q\|X\|_{\Phi} \leqslant q \sup _{n \geqslant 0}\left\|X-X_{n}\right\|_{\Phi} .
$$

holds. Thus, using (1) our inequality is proved with

$$
c_{\Phi}^{(2)}=1 / 2 c_{\Phi}^{\prime \prime} \quad \text { and } \quad C_{\Phi}^{(2)}=q / c_{\Phi}^{\prime}
$$

Ishak and Mogyoródi (see [8, 9] proved the following result:

Theorem 2. Let $\Phi$ be a Young function with finite power $p$ and $\Psi$ denotes its conjugate Young function, not necessarily with finite power. If $X \in H_{\Phi}$ and $Y \in K_{\Phi}$ then the following Fefferman-Garsia type inequality holds

$$
\left|E\left(X_{n} Y_{n}\right)\right| \leqslant c_{\Phi}^{(3)}\left\|X_{n}\right\|_{H_{\ddagger}}\left\|Y_{n}\right\|_{K_{\Psi}}
$$

where $c_{\Phi}^{(3)}$ is a constant depending only on $\Phi$. Further, the limit $\lim _{n \rightarrow+\infty} E\left(X_{n} Y_{n}\right)$ exists and we have

$$
\left|\lim _{n \rightarrow+\infty} E\left(X_{n} Y_{n}\right)\right| \leqslant c_{\Phi}^{(3)}\|X\|_{H_{*}}\|Y\|_{K_{\Psi}} .
$$

Here $X_{n}=E\left(X \mid F_{n}\right)$ and $Y_{n}=E\left(Y \mid F_{n}\right), n \geqslant 0$.

Now, combining the results of Theorems 1 and 2, we have 
TheOREM 3. Let $(\Phi, \Psi)$ be a pair of conjugate Young functions and suppose that both have finite power. If $X \in H_{\Phi}$ and $Y \in Y_{\Psi}$ then

$$
|E(X Y)| \leqslant C\|X\|_{H_{\Psi}}\|Y\|_{H_{\Phi}}
$$

where $E(X Y)=\lim _{n \rightarrow+\infty} E\left(X_{n} Y_{n}\right)$ and $C$ is a constant depending on $\Phi$ and $\Psi$ such that $C=c_{\Phi}^{(3)} c_{\Psi}^{(1)}$.

Proof: Using the result of Theorem 1, we have $Y \in K_{\Psi}$ and

$$
\|Y\|_{K_{\Psi}} \leqslant 1 / c_{\Psi}^{(1)}\|Y\|_{H_{\Psi}}
$$

And using the result of Theorem 2 we have $\lim _{n \rightarrow+\infty} E\left(X_{n} Y_{n}\right)=E(X Y)$ and

$$
|E(X Y)| \leqslant C\|X\|_{H_{\Psi}}\|Y\|_{H_{\Phi}} \text {, where } C=c_{\Phi}^{(3)} / c_{\Psi}^{(1)}
$$

Let $\left(T_{0},\|\cdot\|_{0}\right),\left(T_{1},\|\cdot\|_{1}\right), \ldots$ be a sequence of Banach spaces, and let us define the following Banach spaces

$$
\begin{aligned}
T^{(1)} & =\left\{x=\left(x_{0}, x_{1}, \ldots\right) \in\left(T_{0} \times T_{1} \times \ldots\right):\|x\|^{(1)}=\sum_{n=0}^{\infty}\left\|x_{n}\right\|_{n}<+\infty\right\}, \\
T^{(\infty)} & =\left\{x=\left(x_{1}, x_{1}, \ldots\right) \in\left(T_{0} \times T_{1} \times, \ldots\right):\|x\|^{(\infty)}=\sup _{n \geqslant 0}\left\|x_{n}\right\|_{n}<+\infty\right\},
\end{aligned}
$$

and

$$
T_{0}^{(\infty)}=\left\{x \in T^{(\infty)}: \lim _{n \rightarrow+\infty}\left\|x_{n}\right\|_{n}=0,\|x\|^{(\infty)}=\sup _{n \geqslant 0}\left\|x_{n}\right\|_{n}\right\} .
$$

Now, we formulate the following lemma without proof (see [10]).

LEMMA 3. Let $B_{n}$ be the dual space of $T_{n}, n=0,1,2, \ldots$ Then, the dual space of $\left(T_{0}^{(\infty)},\|\cdot\|^{(\infty)}\right)$ is isomorphic to $\left(B^{(1)},\|\cdot\|^{(1)}\right)$ and isomorphism can be given by the formula

$$
B^{(1)} \ni y \mapsto f_{y}=\sum_{n=0}^{\infty}\left\langle., y_{n}\right\rangle
$$

with $\left\|f_{y}\right\|=\sum_{n=0}^{\infty}\left\|y_{n}\right\|_{n}=\|y\|^{(1)}$ 


\section{MAIN RESUlT}

THEOREM 4. Let $(\Phi, \Psi)$ be a pair of conjugate Young functions and suppose that both of them have finite power. Then, the dual space of the martingale Hardy space $H_{\Phi}$ is the martingale Hardy space $H_{\Psi}$.

Proof: If $Y \in H_{\Psi}$ is fixed and $X$ varies on $H_{\Phi}$ then $\lim _{n \rightarrow+\infty} E\left(X_{n} Y_{n}\right)$ is a continuous and linear functional on $H_{\Phi}$ with norm $\leqslant C\|Y\|_{H_{\Phi}}$. Conversely, suppose $f$ is a continuous and linear functional on $\left(H_{\Phi},\|\cdot\|_{H_{\Phi}}\right)$. Then by Lemma $2, f$ is also continuous with respect to the norm $\sup _{n \geqslant 0}\left\|X-X_{n}\right\|_{\Phi}$. Consider the Banach space $T_{0}^{(\infty)}(\Phi)$ defined by the formula

$$
T_{0}^{(\infty)}(\Phi)=\left\{\lambda=\left(\lambda_{0}, \lambda_{1}, \ldots\right), \lambda_{n} \in L^{\Phi}, n \geqslant 0, \lim _{n \rightarrow+\infty}\left\|\lambda_{n}\right\|=0\right\}
$$

furnished with the norm

$$
\|\lambda\|_{T_{0}^{(\infty)}(\Phi)}=\sup _{n \geqslant 0}\left\|\lambda_{n}\right\|_{\Phi}
$$

Then, the space $\left(H_{\Phi}, \sup _{n \geqslant 0}\left\|X-X_{n}\right\|_{\Phi}\right)$ which can be considered as the set of the sequences

$$
\tilde{X}=\left(X-X_{0}, X-X_{1}, X-X_{2}, \ldots\right), \quad X \in H_{\Phi}
$$

is a subspace of $T_{0}^{(\infty)}(\Phi)$ since $X_{n}$ converges to $X$ almost surely and in $L^{\Phi}$-norm. The continuous and linear functional $f$ given on $\left(H_{\Phi}, \sup _{n \geqslant 0}\left\|X-X_{n}\right\|_{\Phi}\right)$ can be extended to a linear functional $G(\lambda)$ on $T_{0}^{(\infty)}(\Phi)$ with the same norm as that of $f$. This can be done by means of the Hahn-Banach theorem.

Remarking that the dual space of $L^{\Phi}$ is $L^{\Psi}$ and choosing $T_{i}=L^{\Phi}(\Omega, A, P)$, $i=0,1,2, \ldots$, by Lemma 3 there exists a sequence $\left(\sigma_{n}\right)_{n=0}^{\infty}$ of random variables such that $\sigma_{n} \in L^{\Psi}$ with

$$
\sum_{n=0}^{\infty}\left\|\sigma_{n}\right\|_{\Psi} \leqslant\|G\|=\|f\|
$$

We also have

$$
G(\lambda)=\sum_{n=0}^{\infty} E\left(\lambda_{n} \sigma_{n}\right) \quad \text { for all } \lambda \in T_{0}^{(\infty)}(\Phi) .
$$

Consider now the special sequence

$$
\tilde{X}=\left(X-X_{0}, X-X_{1}, X-X_{2}, \ldots, S-X_{n}, \ldots\right)
$$

Putting $\tilde{X}_{n}=\left(X_{n}-X_{0}, X_{n}-X_{1}, \ldots, X_{n}-X_{n-1}, 0,0, \ldots\right)$, we see that

$$
\left\|\tilde{X}-\tilde{X}_{n}\right\|_{T_{0}^{(\infty)}(\Phi)}=\sup _{k \geqslant n}\left\|X-X_{k}\right\|_{\Phi} \rightarrow 0
$$


as $n \rightarrow+\infty$. Consequently,

$$
G(\tilde{X})=\lim _{n \rightarrow+\infty} G\left(\tilde{X}_{n}\right)
$$

Now, easy calculations show that

$$
\begin{aligned}
G\left(\tilde{X}_{n}\right) & =\sum_{i=0}^{n-1} E\left[\left(X_{n}-X_{i}\right) \sigma_{i}\right]=\sum_{i=0}^{n-1} E\left\{\left[E\left(X_{n} \mid F_{n}\right)-E\left(X_{n} \mid F_{i}\right)\right] \sigma_{i}\right\} \\
& =\sum_{i=0}^{n-1} E\left\{X_{n}\left[E\left(\sigma_{i} \mid F_{n}\right)-E\left(\sigma_{i} \mid F_{i}\right)\right]\right\} \\
& =E\left\{X_{n}\left[\sum_{i=0}^{n-1}\left(E\left(\sigma_{i} \mid F_{n}\right)-E\left(\sigma_{i} \mid F_{i}\right)\right)\right]\right\} .
\end{aligned}
$$

Writing

$$
\Delta_{n}=\sum_{i=0}^{n-1}\left[E\left(\sigma_{i} \mid F_{n}\right)-E\left(\sigma_{i} \mid F_{i}\right)\right]
$$

we have

$$
G(\tilde{X})=\lim _{n \rightarrow+\infty} G\left(\tilde{X}_{n}\right)=\lim _{n \rightarrow+\infty} E\left(X_{n} \Delta_{n}\right) .
$$

It is easy to see that $\left(\Delta_{n}, F_{n}\right)$ is a martingale which satisfies

$$
\begin{aligned}
\left\|\Delta_{n}\right\|_{\Psi} & \leqslant \sum_{i=0}^{n-1}\left\|E\left(\sigma_{i} \mid F_{n}\right)-E\left(\sigma_{i} \mid F_{i}\right)\right\|_{\Psi} \leqslant 2 \sum_{i=0}^{n-1}\left\|\sigma_{i}\right\|_{\Psi} \\
& \leqslant 2 \sum_{i=0}^{\infty}\left\|\sigma_{i}\right\|_{\Psi} \leqslant 2\|G\| .
\end{aligned}
$$

This martingale $\left(\Delta_{n}, F_{n}\right)$ is $L^{\Psi}$-bounded. It follows that $\left(\Delta_{n}, F_{n}\right)$ is a regular martingale (see [11]) and there exists a random variable $\Delta \in L^{\Psi}$ such that $\Delta_{n}=E\left(\Delta \mid F_{n}\right)$. We also show that $\Delta \in K_{\Psi}=H_{\Psi}$. This follows from the Doob maximal inequality according to which $\Delta^{*}=\sup _{n \geqslant 0}\left|\Delta_{n}\right| \in L^{\Psi}$, since

$$
\left\|\Delta^{*}\right\|_{\Psi} \leqslant \sup _{n \geqslant 0}\left\|\Delta_{n}\right\| \leqslant 2 p \sum_{n=0}^{\infty}\left\|\sigma_{n}\right\|_{\Psi}<\rightarrow \infty .
$$

This in fact implies that

$$
E\left(\left|\Delta-\Delta_{n-1}\right| \mid F_{n}\right) \leqslant E\left(2 \Delta^{*} \mid F_{n}\right) \quad \text { almost surely for all } n \geqslant 1,
$$

and so $\Delta \in K_{\Psi}$ and $\|\Delta\|_{K_{\Psi}} \leqslant 2\left\|\Delta^{*}\right\|_{\Psi}$. Using the result of Theorem 1 , it follows that $\Delta \in H_{\Psi}$ and

$$
\|\Delta\|_{H_{\Psi}} \leqslant C_{\Psi}^{(1)}\|X\|_{K_{\Psi}} \leqslant 2 C_{\Psi}^{(1)}\left\|\Delta^{*}\right\|_{\Psi}
$$

where $C_{\Psi}^{(1)}$ is a constant depending only on $\Psi$ defined in Theorem 1. This proves our assertion. 


\section{Some REMARKABLE DUALITIES}

As a direct consequence of our main result proved in Section 3, we are now in a position to present the following remarkable dualities:

Theorem 5. If $(\Phi, \Psi)$ is a pair of conjugate Young functions such that both $\Phi$ and $\Psi$ have finite power then:

(i) The martingale space $K_{\Phi}$ is the dual space of the martingale $K_{\Psi}$-space.

(ii) The martingale $H_{a r d y}$ space $H_{\Phi}$ is the dual space of the martingale $K_{\Psi}$ space.

In the special case when $\Phi(x)=x^{p} / p$ and $\Psi(x)=x^{q} / q, 1<p<+\infty$ and $1<$ $q<+\infty$, it follows that the dual of the space $H_{p}$ is the space $K_{q}$, where $1 / p+1 / q=1$, for all the values of $p$ such that $1<p<+\infty$. This can be considered as an extension of Garsia's result (see [2]).

\section{REFERENCES}

[1] C. Fefferman, 'Characterizations of bounded mean oscillations', Bull. Amer. Math. Soc. 77 (1971), 587-588.

[2] A.M. Garsia, Martingale Inequalities (Benjamin Readings, Massachusetts, 1973).

[3] N.L. Bassily and J. Mogyoródi, 'On the $K_{\Phi}$-spaces with general Young function $\Phi$ ', Ann. Univ. Sci. Budapest. Eötvös Sect. Math. XXVII (1985), 205-214.

[4] J. Neveu, Discrete parameter martingales (North-Holland, Amsterdam, 1975).

[5] M.A. Kranoselskii and Ya.B. Rutickii, Convex functions and Orlicz spaces (Noordhoff, Gröningen, 1961).

[6] D.L. Burkholder, B. Davis and R.F. Gundy, 'Integral inequalities for convex functions of operators on martingales', in Proceedings 6th Berkeley symposium on mathematical statistics and probability, pp. 223-240 (University of California Press, 1972).

[7] J. Mogyoródi and F. Móri, 'Necessary and sufficient condition for the maximal inequality of convex Young functions', Acta Sci. Math. 45 (1983), 325-332.

[8] S. Ishak and J. Mogyoródi, 'On the generalization of the Fefferman-Garsia inequality', Stochastic Differential Systems, in Lecture notes in control and information sciences, pp. 85-97 (Springer-Verlag, Berlin, 1981).

[9] S. Ishak and J. Mogyoródi, 'On the $P_{\Phi}$-spaces and the generalization of Hertz' and Fefferman's inequalities I, II and III', Studia Sci. Math. Hungar. 17 (1982), 229-234. 18, pp. 205-210 and 18 (1983), 211-219.

[10] F. Schipp, 'The dual space of the martingale VMO-space', in Proceedings of the 3rd Pannonian symposium on mathematical statistics, pp. 305-311 (Visagrád, Hungary, 1982).

[11] D.L. Burkholder, 'Distribution function inequalities for martingales', Ann. Prob. 1 (1973), 19-42. 
Department of Mathematics American University of Cairo P.O. Box 2511

Cairo

Egypt
The Institute of Statistical Studies

Cairo University

Cairo

Egypt 